
Herausgegeben von

Dr. Klaus Becker-Berke 


\section{MVS/ESA JCL}

Einführung in die Praxis

von

Michael Winter

Deutsche Lufthansa AG

3., korrigierte und aktualisierte Auflage 
Michael Winter sammelte nach seinem Studium mehrjathrige Erfahrung als Anwendungsentwickler in der IBM-Welt in den Umgebungen DOS/VSE und MVS/TSO, bevor er als EDV-Dozent zur Deutschen Lufthansa AG wechselte. Er ist dort verantwortlich fur die Schulung der Programmierer in den Bereichen TSO/ISPF, MVS, JCL und REXX sowie systemnaher Software.

Anschrift:

Michael Winter

Lufthansa Systems GmbH

Am Weiher 24

65451 Kelsterbach

Tel.: +49696966587

Fax: +49696966588

E-Mail: Michael.Winter@dhl.de

\section{Die Deutsche Bibliothek - CIP-Einheitsaufnahme}

\section{Winter, Michael:}

MVS ESA JCL : Einfuhrung in die Praxis / von Michael Winter.

[Hrsg. von Klaus Becker-Berke.]. - 3., korrigierte und aktualisierte

Aufl. - München ; Wien : Oldenbourg, 1999

ISBN 3-486-25058-2

(C) 1999 Oldenbourg Wissenschaftsverlag GmbH

Rosenheimer Straße 145, D-81671 München

Telefon: (089) 45051-0, Internet: http://www.oldenbourg.de

Das Werk einschließlich aller Abbildungen ist urheberrechtlich geschutzt. Jede Verwertung außerhalb der Grenzen des Urheberrechtsgesetzes ist ohne Zustimmung des Verlages unzulässig und strafbar. Das gilt insbesondere für Vervielfăltigungen, Übersetzungen, Mikroverfilmungen und die Einspeicherung und Bearbeitung in elektronischen Systemen.

Lektorat: Birgit Zoglmeier

Herstellung: Rainer Hartl

Umschlagkonzeption: Kraxenberger Kommunikationshaus, München

Gedruckt auf säure- und chlorfreiem Papier

Gesamtherstellung: R. Oldenbourg Graphische Betriebe $\mathrm{GmbH}$, München 